\title{
(Lepidoptera:Gelechiidae) Tuta absoluta المكافحة الحيوية لعثة الطماطة
}

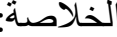

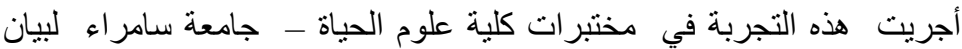

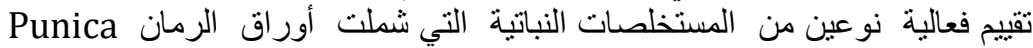

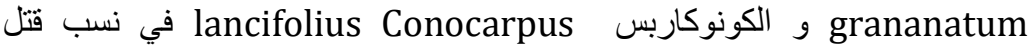

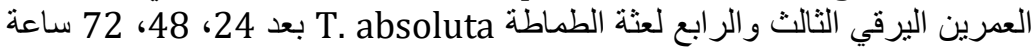

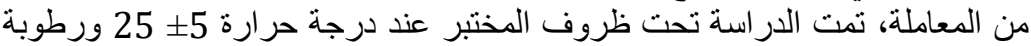

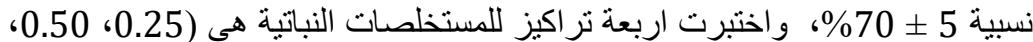

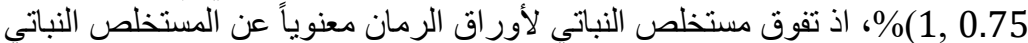

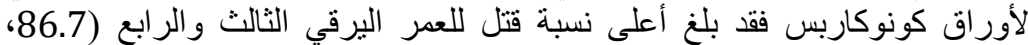

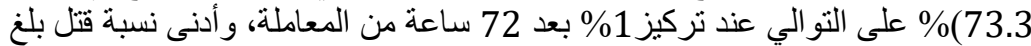

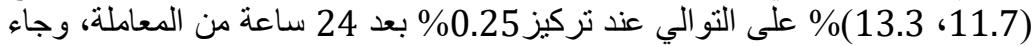

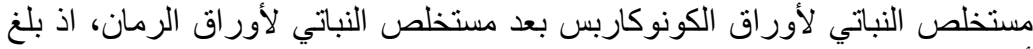

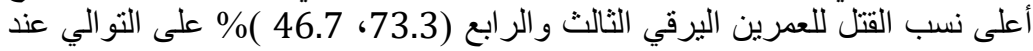

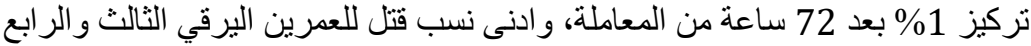

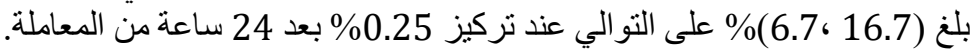

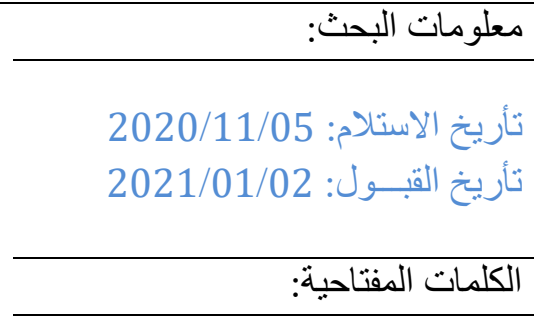

عثة الطعاطة، Tuta absoluta , lancifolius Conocarpus Punica grananatum

يعد محصول الطماطة ئم Solanum Lycopericum من المحاصيل الاكثر شيو عا و المهمة على المستويين المحلي و العالمي وتزرع على نطاق و اسع لكثير من بلدان العالم وهي من محاصيل الاساسية في التغذية عند معظم [1,2] . وتحتل مركزا

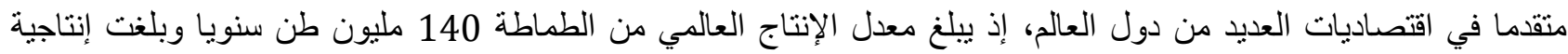
محافظة صلاح الدين 273,764 طن عام 2011 4]2013. يصاب محصول الطماطة في العالم بالعديد من الآفات الزراعية المرضية والحشرية وغير الحشرية، وتعد عثة الطماطة T. absoluta الآفة الرئيسية لمحصول الطماطة والعامل المحدد لإنتاجها

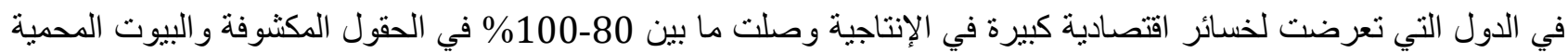
[5]. تعد امريكا الجنوبية الموطن الأصلي و التي أصبحت آفة رئيسية فيها منذ عام 1960 انتقلت إلى أوربا ثم وصلت الى منئية

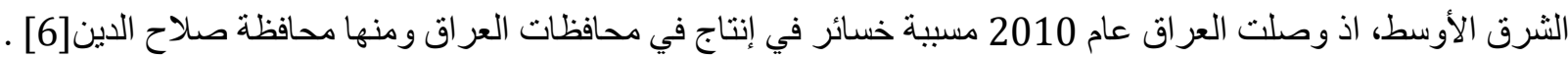

إن الانتشار السريع لعثة الطماطة T. absoluta واستمرار انتقالها بين دول العالم المنتجة للطماطة يعد أعظم تهديد

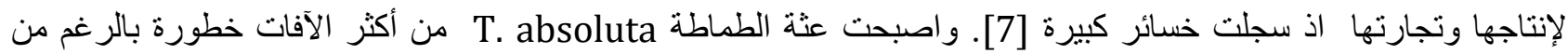

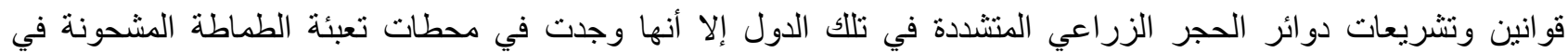

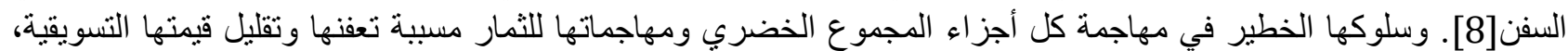

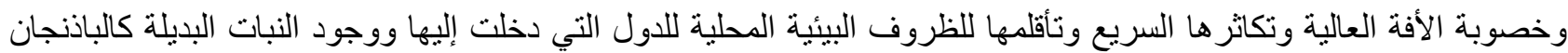
و الفلفل و البطاطا، كل هذا يدفعنا إلى إجر اء بحوث تطبيقية مستمرة ومعمقة لدراسة ديناميكينها في النظام البيئي الزر اعي لأجل الناليان 
إيجاد أفضل الطر ائق في مكافحتها ضمن بر امج أدارة الآفة المتكاملة IPM [9]. ادى الاستعمال المتكرر والغير صحيح و العشوائي

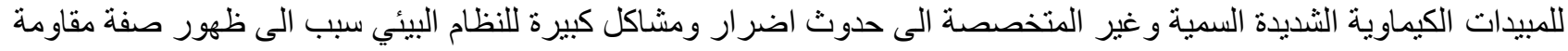

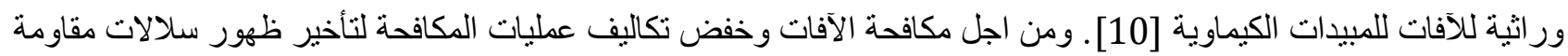

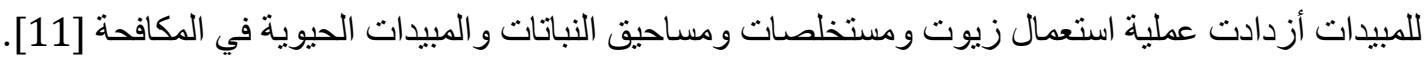

المواد وطرائق العمل

1.تثخيص الحشرة

جمعت عثة الطماطة T. absoluta من أور اق طماطة مصابة بأعمار المختلفة للحشرة من نباتات مصابة بالعثة في البيوت البلاستيكية لمز ارع طماطة مصابة في مدينة سامر اء، حفظت العينات في أطباق بتري 9 سم وثم ارسلت لتشخيص التشات الحشرة

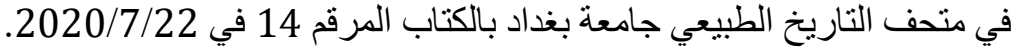

2. 2 تهيئة المبيدات الاحيائية جمعت اجز اء النباتات و التي شملت أوراق نبات الرمان وأو أوراق نبات الكونوكاربس كما في الثكل1 من بستان في مدينة سامراء، عزلت الاوراق السليمة والخالية من الاصدابات او أي اضرار اخرى، نظفت الاوراق جيدا ووضعت على على شكل طبقة

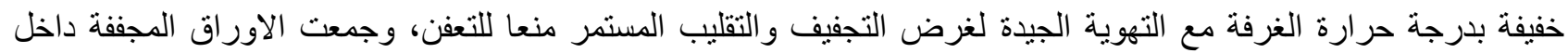
اكياس لحفظها في ظروف ملائمة خالية من الرطوبة لحين طحنها واستعمالها في التجارب الخاصة بمحاور الدراسة ثم طحنت

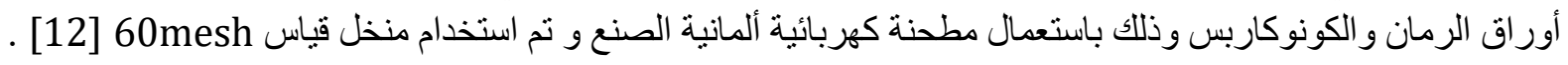
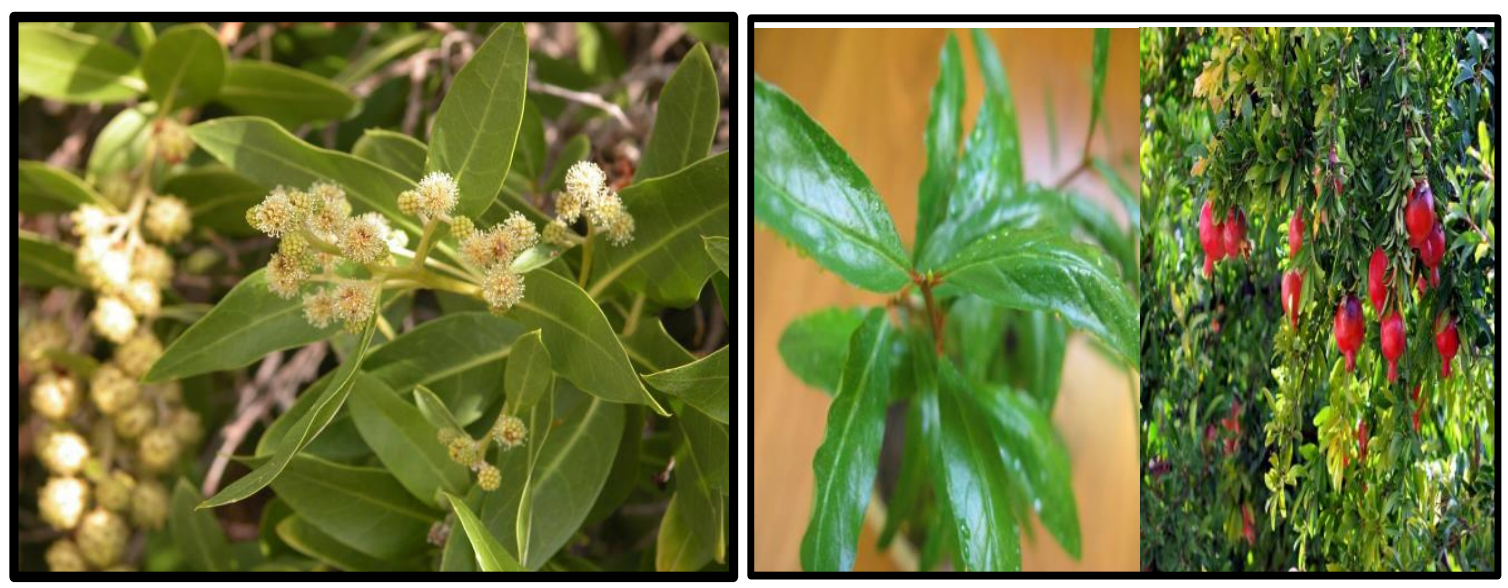

شكل 1: أور اق نبات الرمان P. granatum و نبات الكونوكاربس C. lancifolius.

3. تحضير المستخلصات النباتية

تم تحضير المستخلصات النباتية في مختبر ات قسم علوم الحياة ـكلية التربية -جامعة سامر اء وذللك وتم استعمال كحول

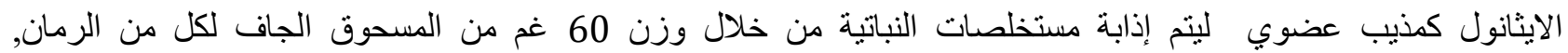
الكونوكاربس ويكون كل على حده في وعاء الاستخلاص Extraction Thamble، لجهاز الاستخلاص extracter المستخلص تحت درجة حر ارة 40 م وضغط واطئ ويستمر التسخين لحين زوال اللون النباتي الغامق واستغرقت هذه العملية من

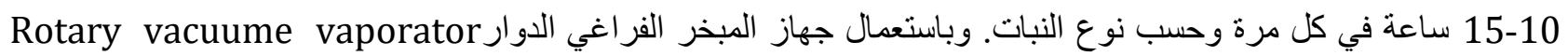

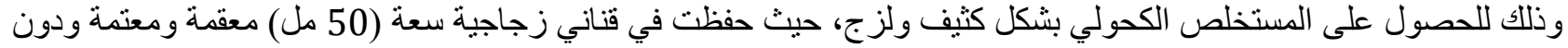

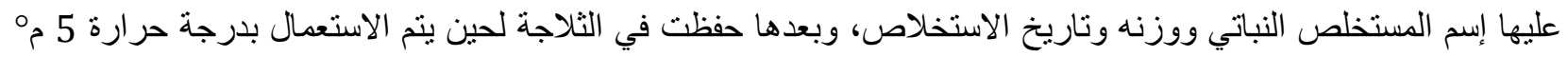

4. تقييم فاعلية المبيدات الاحيائية ضد يرقات عثة الطماطة T. absoluta مختبريا

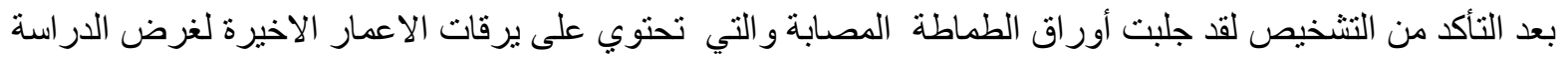

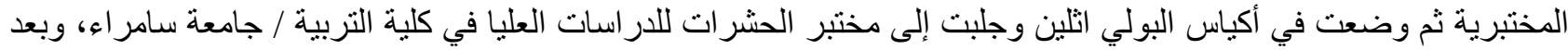

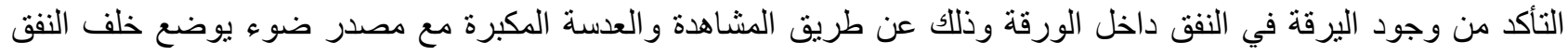

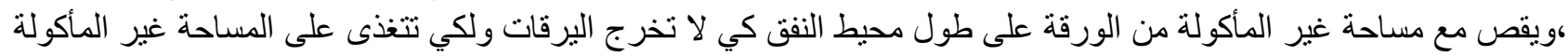
في مدة اخذ القراءات بواسطة مقص يعقم كل مرة تقص فيها ورقة بعدها وضعت كل 10 يرقات من اوراق الطماطة كل قطعة 
تحتوي يرقة في النفق ،في الطبق البتري بداخله ورقة ترشيح [14]. واستعمل القطن مبلل بالماء ووضع على الإنى الاور اق للمحافظة

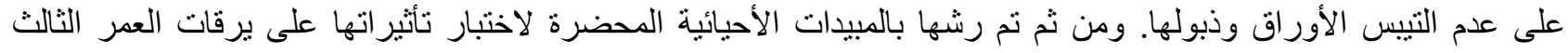

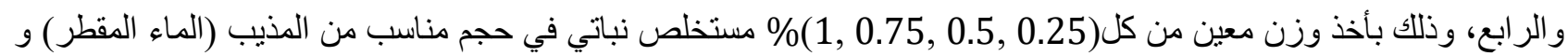

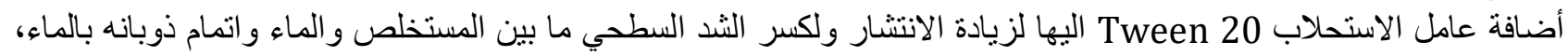

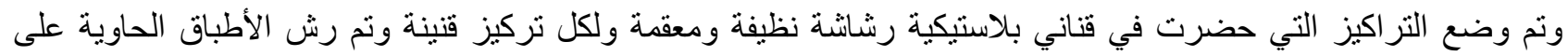

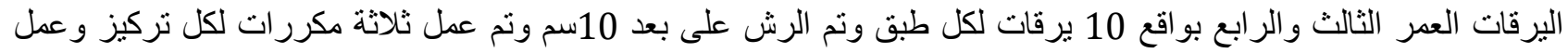

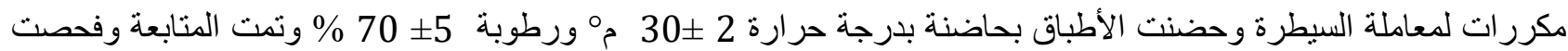

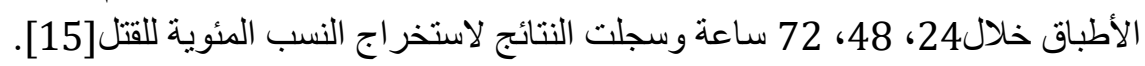

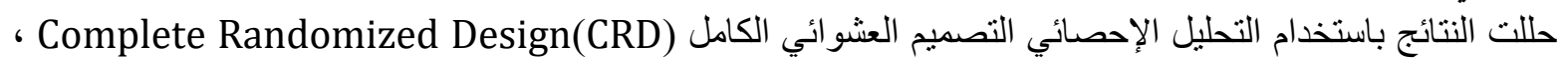

\section{التحليل الإحصائي} ولمقارنة النتائج استخدم اختبار دنكن ذي المدى المتعدد عند مستوى احتمالية 0.05 s p [16].

النتائج والمناقشة

تأثثر المستخلصات النباتية في العمرين الثالث و الر ابع ليرقات عثة الطماطة T. absoluta مختبرياً:-

1. ت تأثير المستخلص النباتي لأوراق الرمان بعد 24 ساعة من المعاملة:

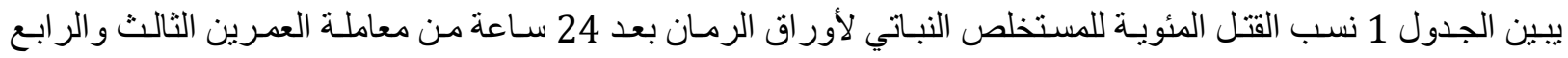

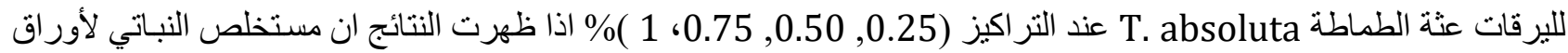

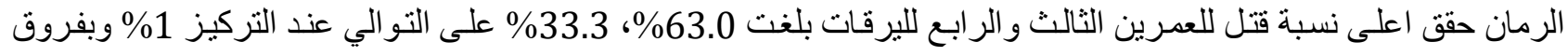

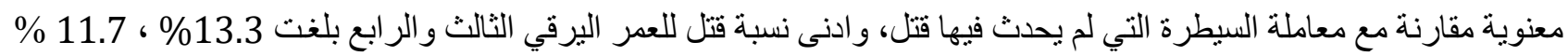

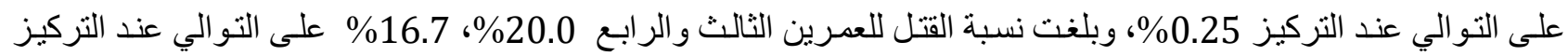

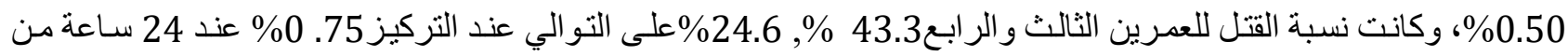
المعاملة، وتبين النتائج وجود فرق معنوية في نسب القتل مقارنة بمعاملة السيطرة التي لم يحدث فيها قتل.

جدول 1: نسب القتل المئوية للعمرين الثالث و الر ابع لعثة الطماطة T. absoluta لمستخلص النباتي لأوراق الرمان بعد 24 ساعة من المعاملة.

\begin{tabular}{|c|c|c|c|c|c|c|c|}
\hline \multirow[b]{2}{*}{ فتوسط القتل } & \multirow[b]{2}{*}{ فتوسط الطور } & \multicolumn{4}{|c|}{ التراكيز المستعملة غم/ 100 مل } & \multirow[b]{2}{*}{ الطور اليرقي } & \multirow[b]{2}{*}{ 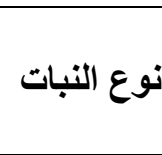 } \\
\hline & & 1 & المئوية & ن & 0.25 & & \\
\hline \multirow{3}{*}{$\begin{array}{c}27.1 \\
\mathrm{~A}\end{array}$} & $\begin{array}{c}34.9 \\
\mathrm{~A}\end{array}$ & 63.0 & 43.3 & 20.0 & 13.3 & 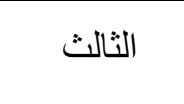 & \multirow{2}{*}{ الرمان } \\
\hline & $\begin{array}{c}21.5 \\
\mathrm{D}\end{array}$ & 33.3 & 24.6 & 16.7 & 11.7 & 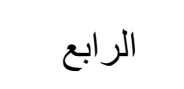 & \\
\hline & & $\begin{array}{c}48.3 \\
\mathrm{a}\end{array}$ & $\begin{array}{c}33.9 \\
\mathrm{~b}\end{array}$ & $\begin{array}{c}18.5 \\
c\end{array}$ & $\begin{array}{c}12.5 \\
\mathrm{D}\end{array}$ & \multicolumn{2}{|c|}{ منوسط القتل في تركيز الرمان } \\
\hline \multirow{2}{*}{$\begin{array}{c}0.0 \\
\mathrm{D}\end{array}$} & $\begin{array}{c}0.0 \\
G\end{array}$ & 0.0 & 0.0 & 0.0 & 0.0 & 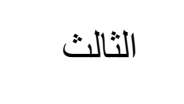 & \multirow[b]{2}{*}{ Control } \\
\hline & $\begin{array}{c}0.0 \\
\mathrm{G}\end{array}$ & 0.0 & 0.0 & 0.0 & 0.0 & 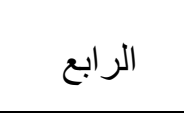 & \\
\hline
\end{tabular}

اتفقت نتائج در استتا مع دراسة[17] حول تأثثر المستخلص الكحولي و المائي لأوراق الرمان وتأثثره على التركيب النسيجي التئي

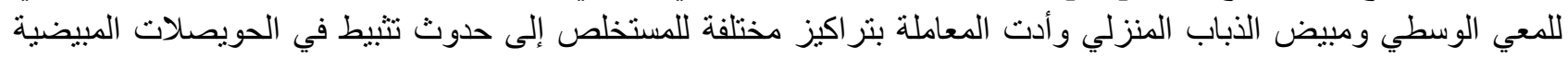

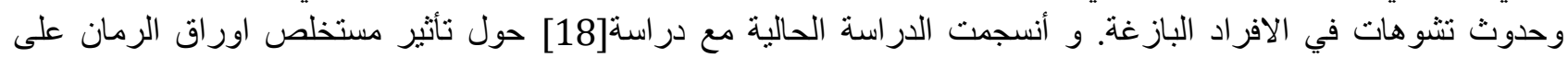

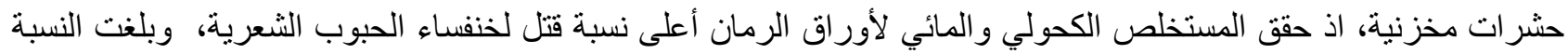
100 \% في التركيز\%\% \% \%بعد 24 من ساعة من المعاملة . 
2. تأثير المستخلص النباتي لأور اق الرمان بعد 48 ساعة من المعاملة:

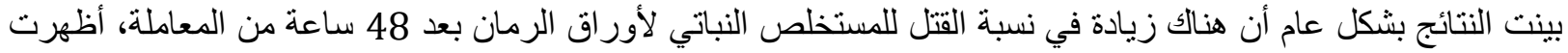

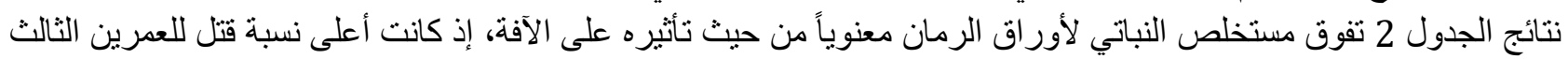

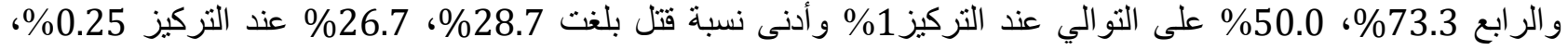

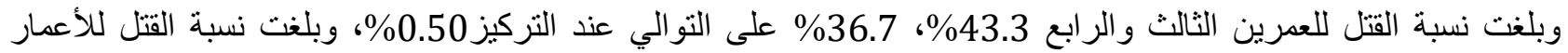
اليرقية 49.3 \%، 43.3\% عند التركيز 0.75\%، وبفروق معنوي \% \% \%

جدول 2: نسب القتل المئوية للعمرين الثالث و الر ابع لعثة الطماطة T. absoluta لمستخلص النباتي لأور اق الرمان بعد 48

ساعة من المعاملة.

\begin{tabular}{|c|c|c|c|c|c|c|c|}
\hline متوسط & متوسط & \multicolumn{4}{|c|}{ التراكيز المستعملة غم/ 100 مل } & \multirow{3}{*}{ الطور اليرقي } & \multirow{3}{*}{ نوع النبات } \\
\hline القتل في & القتل في & 1 & 0.75 & 0.50 & 0.25 & & \\
\hline النوع & الطور & \multicolumn{4}{|c|}{ نسبة القتل المئوية } & & \\
\hline \multirow{6}{*}{$\begin{array}{c}43.9 \\
\text { A }\end{array}$} & 48.6 & 723 & 402 & 432 & 287 & 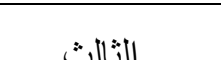 & \multirow{4}{*}{ اور اق الرمان } \\
\hline & $\mathrm{D}$ & 10.0 & 49.5 & 40.5 & 20.1 & \multirow{3}{*}{ الر ابع } & \\
\hline & 39.2 & 500 & 122 & 267 & 267 & & \\
\hline & $\mathrm{B}$ & 30.0 & 45.5 & 30.1 & 20.1 & & \\
\hline & & 61.1 & 46.3 & 40 & 27.7 & \multirow{2}{*}{\multicolumn{2}{|c|}{ متوسط القتل في تركيز الرمان }} \\
\hline & & $\mathrm{a}$ & $\mathrm{b}$ & C & $\mathrm{D}$ & & \\
\hline \multirow{3}{*}{$\begin{array}{c}0.0 \\
D\end{array}$} & 0.0 & 00 & 00 & 00 & 00 & الثالث & \multirow{3}{*}{ Control } \\
\hline & $\mathrm{G}$ & & & & & & \\
\hline & $\begin{array}{c}0.0 \\
G\end{array}$ & 0.0 & 0.0 & 0.0 & 0.0 & 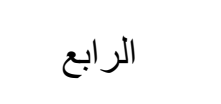 & \\
\hline
\end{tabular}

يمكن القول عن زيادة التركيز يؤدي إلى ازدياد نسب القتل يعود الى كمية المركبات الفعالة الموجود في المستخلص الكحولي

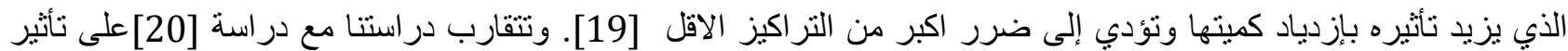

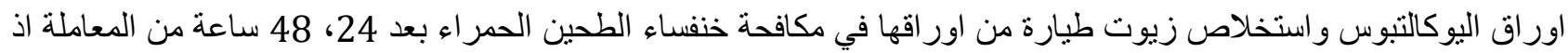
حقق نسب قتل بلغت 86.2\% عند اعلى تركيز 8\% وارتفعت بعد مرور 48 ساعة من المعاملة إلى و ادنى نسبة قتل كانت لأدنى تركيز واشار في در استه إلى ان زيادة التركيز ومدة التعريض يؤدي إلى زئى زيادة نسب قتل الحشرة .

3. تأثير المستخلص النباتي لأوراق الرمان بعد 72 ساعة من المعاملة:

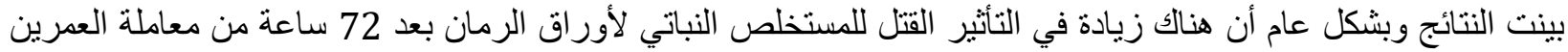

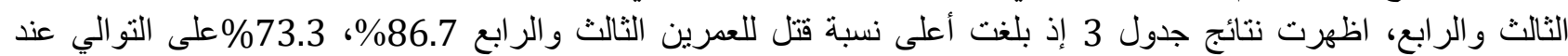
التركيز 1\%، وادنى نسبة قتل للأعمار اليرقية بلغت 46.7\%, 36.7\%على التوالي عند التركيز 0.25\%، وكانت نسبة القتل

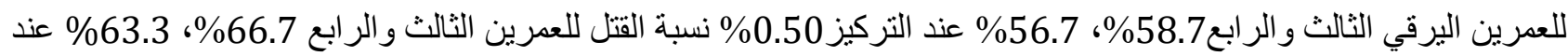

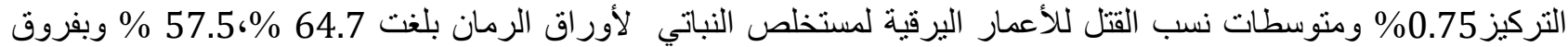

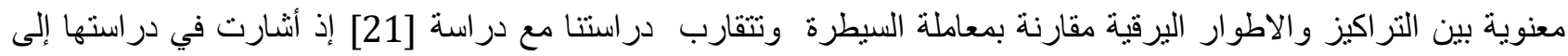

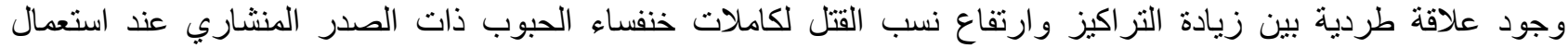

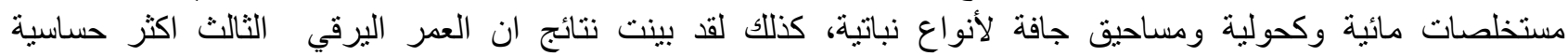
بالمستخلصات النباتية. 
جدول 3: نسب التتل المئوية للعمرين اليرقي الثالث و الر ابع لعثة الطماطة T. absoluta لمستخلص النباتي اوراق الرمان بعد 72

\begin{tabular}{|c|c|c|c|c|c|c|c|}
\hline متوسط & متوسط & \multicolumn{4}{|c|}{ التراكيز المستعملة غم/ 100 مل } & \multirow{3}{*}{ الطور اليرقي } & \multirow{3}{*}{ نوع النبات } \\
\hline القتل في & القتل في & 1 & 0.75 & 0.50 & 0.25 & & \\
\hline النوع & الطور & & المئوية & 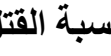 & & & \\
\hline \multirow{6}{*}{$\begin{array}{c}61.1 \\
\mathrm{~A}\end{array}$} & 64.7 & 86.7 & 66.7 & 58.7 & 46.7 & الثالث & \multirow{4}{*}{ اوراق الرمان } \\
\hline & $\mathrm{D}$ & & & & & & \\
\hline & 57.5 & 73.3 & 63.3 & 56.7 & 36.7 & الر ابع & \\
\hline & A & & & & & & \\
\hline & & 80.0 & 65.0 & 57.7 & 41.7 & \multirow{2}{*}{\multicolumn{2}{|c|}{ متوسط القتل في الرمان }} \\
\hline & & a & $\mathrm{b}$ & $\mathrm{C}$ & $\mathrm{d}$ & & \\
\hline \multirow{3}{*}{$\begin{array}{c}0.0 \\
D\end{array}$} & 0.0 & 0.0 & 0.0 & 0.0 & 0.0 & الثالث & \multirow{3}{*}{ Control } \\
\hline & $\mathrm{H}$ & & & & & & \\
\hline & $\begin{array}{c}0.0 \\
\mathrm{H}\end{array}$ & 0.0 & 0.0 & 0.0 & 0.0 & 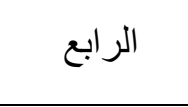 & \\
\hline
\end{tabular}

4. تأثير المستخلص النباتي لأوراق الكونوكاربس بعد 24 ساعة من المعاملة:

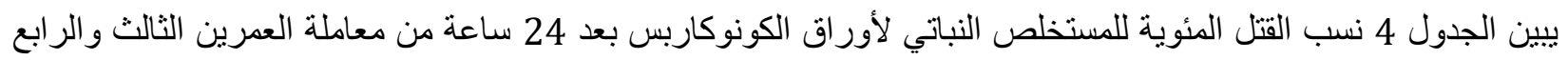

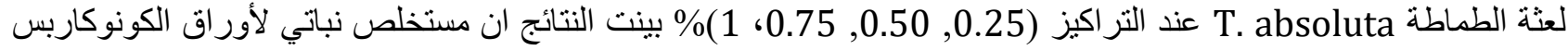

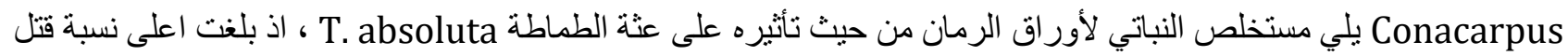

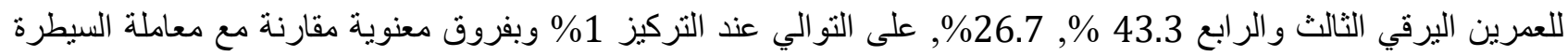

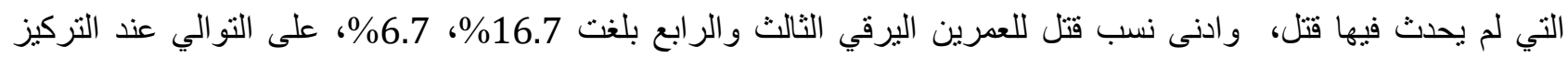

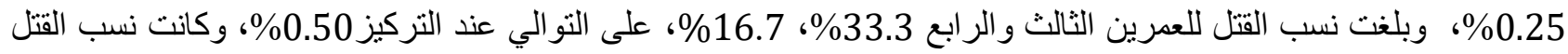

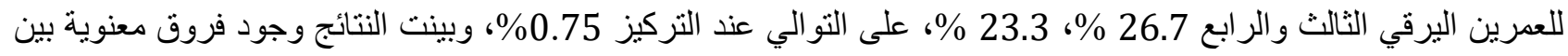

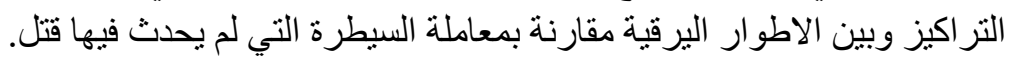

جدول 4: نسب التتل المئوية للعمرين اليرقي الثالث و الر ابع لعثة الطماطة T. absoluta لمستخلص النباتي لأور اق الكونوكاربس بعد 24 ساعة من المعاملة.

\begin{tabular}{|c|c|c|c|c|c|c|c|}
\hline متوسط & متوسط & \multicolumn{4}{|c|}{ التراكيز المستعملة غم/ 100 مل } & \multirow{3}{*}{ الطور اليرقي } & \multirow{3}{*}{ نوع النبات } \\
\hline القتل في & القتل في & 1 & 0.75 & 0.50 & 0.25 & & \\
\hline النوع النوع & الطور & \multicolumn{4}{|c|}{ نسبة القتل المئوية } & & \\
\hline \multirow[b]{2}{*}{24.2} & 30.0 & 122 & 267 & 222 & 167 & $\therefore, 14$ & \multirow{3}{*}{ الكونوكاربس اق } \\
\hline & $\mathrm{C}$ & 43.3 & 20.1 & 33.3 & 10.1 & القاس & \\
\hline \multirow[t]{3}{*}{$\mathrm{B}$} & $\begin{array}{c}18.4 \\
B\end{array}$ & 26.7 & 23.3 & 16.7 & 6.7 & 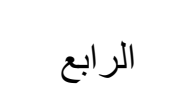 & \\
\hline & & 35 & 25 & 25 & 11.7 & \multicolumn{2}{|c|}{ متوسط القتل في تركيز } \\
\hline & & $\mathrm{a}$ & $\mathrm{b}$ & $\mathrm{C}$ & $\mathrm{d}$ & \multicolumn{2}{|c|}{ الكونوكاربس } \\
\hline \multirow{3}{*}{$\begin{array}{c}0.0 \\
\mathrm{D}\end{array}$} & 0.0 & 0.0 & 0.0 & 0.0 & 0.0 & 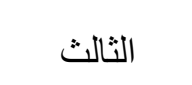 & \multirow{3}{*}{ Control } \\
\hline & $\begin{array}{l}\mathrm{G} \\
0.0\end{array}$ & & & & & & \\
\hline & $\mathrm{G}$ & 0.0 & 0.0 & 0.0 & 0.0 & 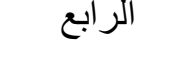 & \\
\hline
\end{tabular}

وان سبب تأثير المستخلص الكحولي لأوراق الكونوكاربس على عثة الطماطة T. absoluta هو بسبب وجود مركبات سامة ذائبة في الكحول اثرت على الوظائف الحيوية الداخلية للحشرة بما ادى إلى توقفها ثم الموت، من هذه المركبات هي 
القلويدات و الكلايوسيدات والتانينات و الفينو لات وكذلك لوجود عن المواد الدباغية التي تحتويها أور اق الكونوكاربس و التي تكون

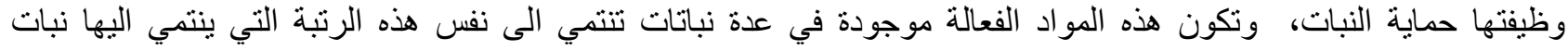

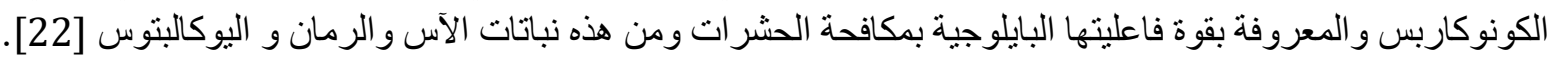

5. تأثثير المستخلص النباتي لأوراق الكونوكاربس بعد 48 ساعة من المعاملة:توضح نتائج الجدول 5 أن المستخلص الكحولي النباتي لأوراق الكونوكاربس يلي مستخلص النباتي لأوراق التاقي الرمان في تأثيره

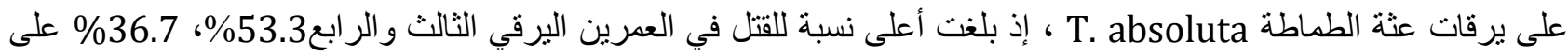

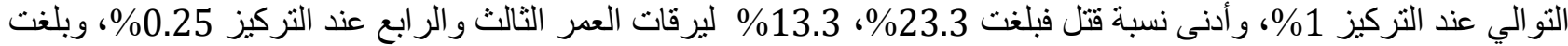

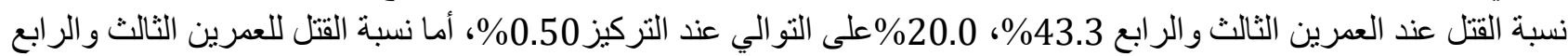

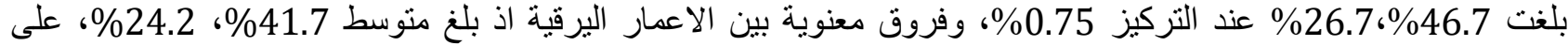
التو الي مقارنة مع معاملة السيطرة التي لم يحدث فيها قتل .

جدول 5: نسب القتل المئوية للعمرين الثالث و الر ابع لعثة الطماطة T. absoluta لمستخلص النباتي لأور اق الكونوكاربس بعد

48 ساعة من المعاملة.

\begin{tabular}{|c|c|c|c|c|c|c|c|}
\hline متوسط & متوسط & \multicolumn{4}{|c|}{ التراكيز المستعملة غم/ 100 مل } & \multirow{3}{*}{ الطور اليرقي } & \multirow{3}{*}{ نوع النبات } \\
\hline القتل في & القتل في & 1 & 0.75 & 0.50 & 0.25 & & \\
\hline النوع & الطور & \multicolumn{4}{|c|}{ نسبة القتل المئوية } & & \\
\hline \multirow{6}{*}{$\begin{array}{c}32.1 \\
\mathrm{~B}\end{array}$} & 41.7 & $52 ?$ & 167 & 127 & 727 & & \multirow{4}{*}{ الكونوكارب } \\
\hline & A & 53.3 & 46.1 & 43.3 & 23.3 & النالت & \\
\hline & 24.2 & 267 & 267 & 200 & 122 & 211 & \\
\hline & $\mathrm{C}$ & 36.1 & 20.1 & 20.0 & 13.3 & الربع & \\
\hline & & 45 & 36.7 & 31.1 & 18.3 & \multirow{2}{*}{\multicolumn{2}{|c|}{ منوسط القتل في تركيز }} \\
\hline & & $\mathrm{a}$ & $\mathrm{b}$ & c & $\mathrm{D}$ & & \\
\hline \multirow{3}{*}{$\begin{array}{c}0.0 \\
\mathrm{D}\end{array}$} & 0.0 & 0.0 & 0.0 & 0.0 & 0.0 & 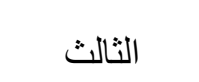 & \multirow{3}{*}{ Control } \\
\hline & G & & & & & & \\
\hline & $\begin{array}{c}0.0 \\
G\end{array}$ & 0.0 & 0.0 & 0.0 & 0.0 & 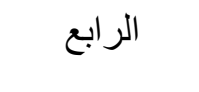 & \\
\hline
\end{tabular}

وتتقارب نتائج هذه الدراسة الحالية دع دراسة [23] حول تقييم الأثر القاتل للمستخلص المائي لأوراق نبات الكونوكاربس

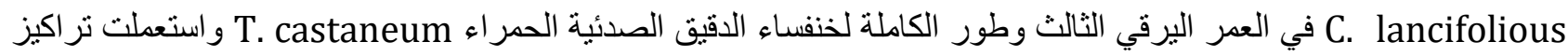

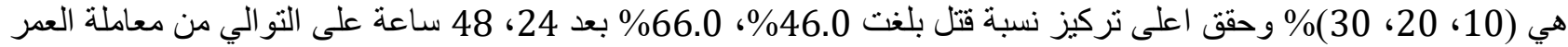

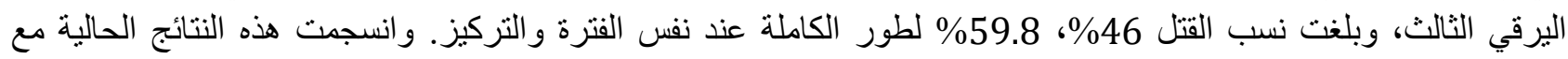

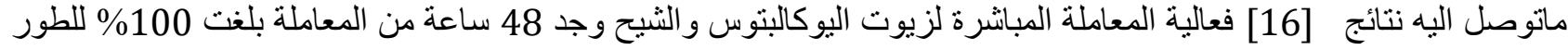

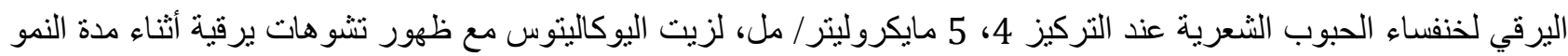
و التطور. وبشكل عام ينم تفسير ان هنالك علاقة طردية بين زيادة التر اكيز ونسب القتل المئوية، وقد يعود السبب إلى التباين في لئي

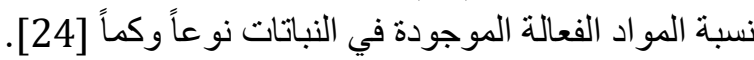

6. نأثير المستخلص النباتي لأوراق الكونوكاربس بعد 72 ساعة من المعاملة: بينت نتائج جدول (6) إلى أن مستخلص النباتي لأور اق الكونوكاربس قد حقق نسب قتل للعدرين اليرقي الثالث و الر ابع بلغت

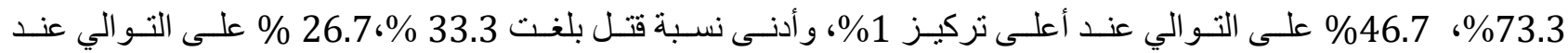

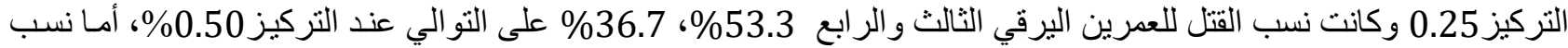

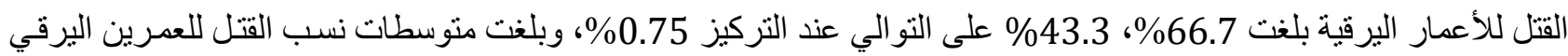
الثالث والر ابع 56.6\%، 40.0\%، عند 72 ساعة بعد المعاملة وبفرق معنوي. 
جدول 6: نسب القتل المئوية للعمرين الثالث و الر ابع لعثة الطماطة T. absoluta لمستخلص النباتي لأور اق الكونوكاربس بعد

2 72 ساعة من المعاملة.

\begin{tabular}{|c|c|c|c|c|c|c|c|}
\hline متوسط & متوسط & \multicolumn{4}{|c|}{ التراكيز المستعملة غم/ 100 مل } & \multirow{3}{*}{ الطور اليرقي } & \multirow{3}{*}{ نوع النبات } \\
\hline القتل في & القتل في & 1 & 0.75 & 0.50 & 0.25 & & \\
\hline النوع & الطور & \multicolumn{4}{|c|}{ نسبة القتل المئوية } & & \\
\hline \multirow{6}{*}{$\begin{array}{c}48.3 \\
\text { B }\end{array}$} & 56.6 & \multirow{2}{*}{73.3} & \multirow{2}{*}{66.7} & \multirow{2}{*}{53.3} & \multirow{2}{*}{33.3} & \multirow{2}{*}{ 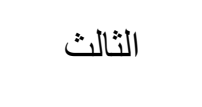 } & \\
\hline & $\mathrm{A}$ & & & & & & 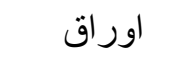 \\
\hline & 40.0 & \multirow{2}{*}{46.7} & \multirow{2}{*}{43.3} & \multirow{2}{*}{36.7} & \multirow{2}{*}{26.7} & \multirow{2}{*}{ 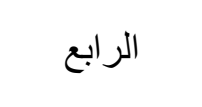 } & \multirow{2}{*}{ الكونوكاربس } \\
\hline & $\mathrm{C}$ & & & & & & \\
\hline & & 60 & 55 & 45 & 30 & \multirow{2}{*}{\multicolumn{2}{|c|}{ متوسط القتل في الكونوكاربس }} \\
\hline & & $\mathrm{a}$ & $\mathrm{b}$ & c & $\mathrm{D}$ & & \\
\hline \multirow{3}{*}{$\begin{array}{c}0.0 \\
D\end{array}$} & 0.0 & 0.0 & 0.0 & 0.0 & 0.0 & 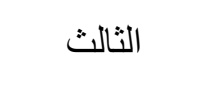 & \multirow{3}{*}{ Control } \\
\hline & 0.0 & \multirow[b]{2}{*}{0.0} & \multirow[b]{2}{*}{0.0} & & & & \\
\hline & $\mathrm{H}$ & & & 0.0 & 0.0 & 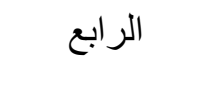 & \\
\hline
\end{tabular}

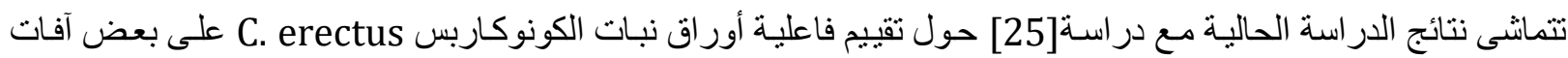

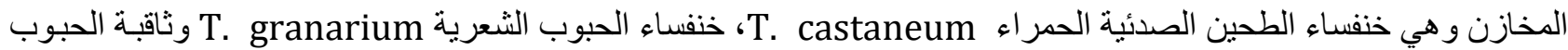

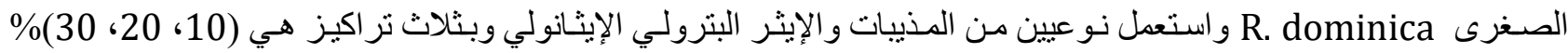
وحقت نسبة قتل للخنفساء الحبوب الثعرية اذ بلغت نسبة القتل لمستخلص الايثر البترولي 14.46\%، 51.0\%؛ 89.44\% \% على

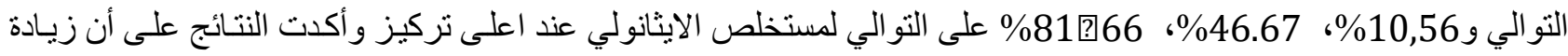
الفترة الزمنية للتعريض تزيد من قتل الحشرات.

\section{References}

1. Gottfridsson, J. (2012). Vårvetesorters effektivitet i användningen av kväve under den huvudsakliga tillväxtperioden.

2. Zhu, Z., Zhang, Y., Liu, J., Chen, Y., \& Zhang, X. (2018). Exploring the effects of selenium treatment on the nutritional quality of tomato fruit. Food chemistry, 252, 9-15.

3. الجهاز المركزي للإحصاء وتكنولوجيا المعلومات .(2017). المحاصيل الثأنوية والخضر أوات. مديرية الإحصاء الزراعي،

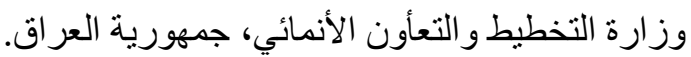
4. حارث برهان الدين عبدالرحمن. (2011). تأثثير نظام الري ومصدر التغذية في النمو الانتاجية والاضرار الفسلجية و المحتوى المعدني لهجينين من الطماطة.

5. Ferrara, F. A., Vilela, E. F., Jham, G. N., Eiras, Á. E., Picanço, M. C., Attygalle, A. B., ... \& Meinwald, J. (2001). Evaluation of the synthetic major component of the sex pheromone of Tuta absoluta (Meyrick) (Lepidoptera: Gelechiidae). Journal of chemical ecology, 27(5), 907-917.

6. Al-Jboory, I. J., Katbeh-Bader, A., \& Al-Zaidi, S. (2012). First observation and identification of some natural enemies collected from heavily infested tomato by Tuta absoluta (Meyrick)(Lepidoptera: Gelechiidae) in Jordan. Middle-East Journal of Scientific Research, 11(4), 435-438.

7. عمار كريم خضير الجسمان. (2018). تقويم بعض العوامل الأحيائية و الكيميائية في مكافحة حشرة حفار اوراق الطماطة (Meyrick,1917) Tuta absoluta (Lepidoptera:Gelechiidae) و العلوم الهندسية، 16(1): 167-159. 
8. EPPO. (2010). First report of Tuta absoluta in Cyprus (2010/003). EPPO Reporting Services 1(003).Accessed February 22, 2010.

http://www.eppo.orgPUBLICATIONS/reporting/reporting service.htm

9. Gupta, A. (2016). Journal of Entomology and Zoology Studies.

10. Riaz, T., Shakoori, F.R. and Ali, S.S., (2018). Phosphineinduced alterations in microsomal enzymes of a stored grain pest Trogoderma granarium collected from godowns of Punjab, Pakistan. Pakistan J. Zoo.,1(50), 291-297.

11. Estay, P. (2000). Polilla del tomate Tuta absoluta (Meyrick). Instituto de Investigationes Agropecuarias, Centro Regional de Investigacion La Platina, Ministerio de Agricultura Santiago Chile.

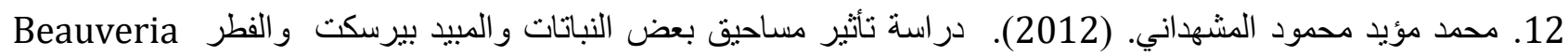
bassiana تكريت.

13. Islam, B. N. (1983). Pesticide action of neem and certain indigenous plant proc. 2nd. Int. Neem. Conf. Ranischolzhousen, 2(4),263-290.

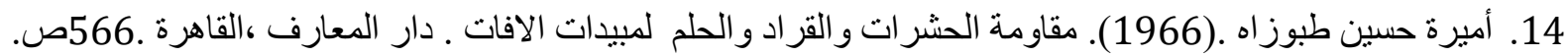

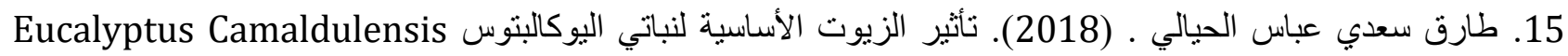

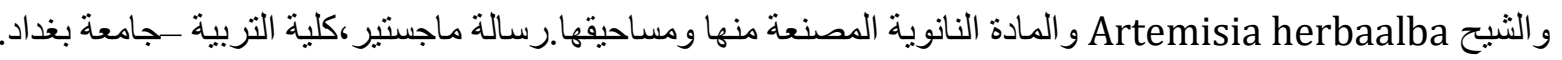

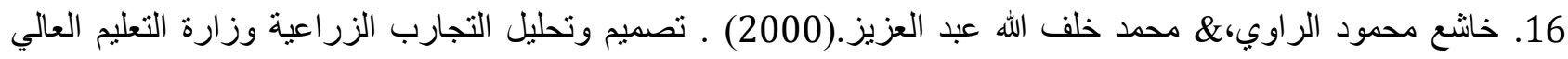

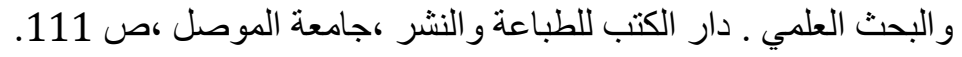
17. صفاء محمد محمود. (2010). تأثير المستخلص المائي لأوراق الرمان النان على التركيب النسجي للمعي الوسطي ومبايض الذباب المنزلي Musca domestica L. مجلة تكريت للعلوم الصرفة, 15(2020), 174-180.

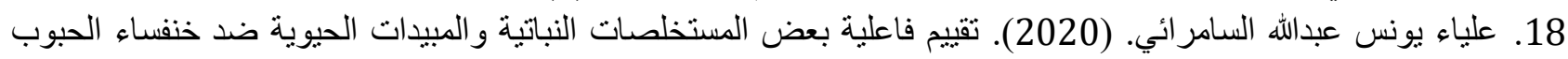
الثعرية (الخابر I ) Trogoderma granarium. رسالة الماجستير ، كلية التربية - جامعة سامر اء.

19. Yousif, R. E. H. E. (2016). Evaluation of some plant extracts against adults of the Sawtoothed grain beetle, Oryzaephilus surinamensis (Doctoral dissertation, Sudan University of Science and Technology).

20. منال ضياء محمد الزبيدي. (2016). تحليل بعض الزيوت النباتية الطيارة والثابتة لبعض المستخلصات النباتية ودراسة تأثثير ها في خنفساء الطحين الصدئية الحمر اء Tribolium Castaneum (Herbst). النباتـ كلية العلوم- الجامعة المستنصرية.

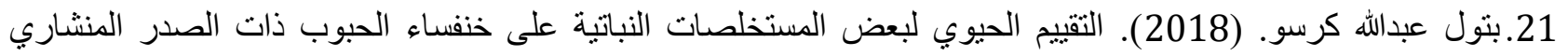

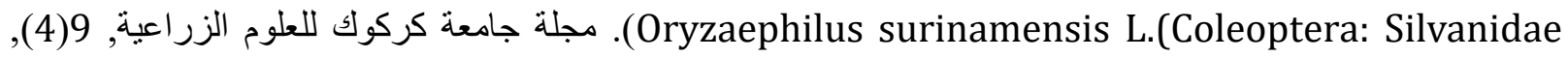
.170-164

22. نداء عبد الله مزبان, \& أ. م. د علي مانع حسين. (2015). دراسة مقارنة لتأثير القلويدات المستخلصة من اوراق نباتي

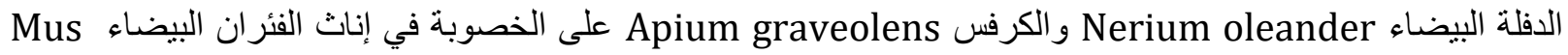
musculus L

23. Salih, D. S. A. (2016). Effect of Leaves Powder Aqueous Extract of some trees against Red flour beetle Tribolium castaneum under Store Conditions (Doctoral dissertation, Sudan University of Science and Technology).

24. Mhemed, A. J. (2011). The efficacy of four seed powders on some biological aspects and mortality of khapra beetle. Iraqi Journal of Agricultural Science, 42(6), 112-123.

25. Asrar, M., Ashraf, N., Gogi, M. D., Hussain, S. M., Zia, K., \& Rasool, B. (2016). TOXICITY AND REPELLENCE OF PLANT OILS AGAINST TRIBOLIUM CASTANEUM (HERBST), RHYZOPERTHA DOMINICA (F.) ANDTROGODERMA GRANARIUM (E.). 


\section{Biological control of tomato moth Tuta absoluta (Lepidoptera: Gelechiidae) in laboratory}

Mareb A. Shawkat ${ }^{1 *}$, Husham Naji Hameed ${ }^{2}$

Department of Life Sciences, Faculty of Education, Samarra University ( maaribalbbasi@gmail.com)

\section{Article Information Abstract}

Received: 05/11/2020

Accepted: 02/01/2021

\begin{tabular}{l}
\hline Keywords: \\
\hline Tuta absoluta, lancifolius \\
Conocarpus, Punica \\
grananatum
\end{tabular}

This experiment was conducted in the laboratories of the College of Life Sciences - University of Samarra to demonstrate the evaluation of the effectiveness of two types of plant extracts, including Punica grananatum leaves and lancifolius Conocarpus, in killing the third and fourth larval ages of T. absoluta after 24, 48 and 72 hours of treatment. The study was conducted under laboratory conditions at a temperature of $5 \pm 25$ and a relative humidity of $5 \pm 70 \%$, and four concentrations of plant extracts were tested $(0.25,0.50,0.75,1) \%$, as the pomegranate leaf extract significantly exceeded the extract Conocarp, it reached the highest percentage of killing For the third and fourth larval ages $(86.7,73.3) \%$, respectively, at a concentration of $1 \%$ after 72 hours of treatment, and the lowest killing percentage was (11.7 and 13.3$) \%$, respectively, at a concentration of $0.25 \%$ after 24 hours of treatment. Conocarpus leaf extract followed an extract Pomegranate leaves, as the highest killing rates for the third and fourth larval ages were (73.3 and 46.7)\%, respectively at a concentration of $1 \%$ after 72 hours of treatment, and the lowest killing rates for the third and fourth larval ages were $(16.7,6.7) \%$ respectively at a concentration of $0.25 \% 24$ hours after the transaction. 